\section{Ein seltener Fall von hyperpyretischem Gelenkrheumatismus ohne nennenswerthe cerebrale Symptome.}

\section{Von Dr. Carl Rosenthal in Berlin.}

Der hyperpyretische Gelenkrheumatismus (Cerebralrheumatismus) ist eine so seltene Erscheinung - es kommen auf etwa 200 Fälle von Polyarthritis rheumatica ein solcher mit hyperpyretischen Erscheinungen - , dass eine jede neue diesbezügliche Beobachtung einiges Interesse zu erwecken imstande ist. Mehr noch muss dies der Fall sein, wenn, wie in folgendem, das Krankheitsbild noch mehrfache und nicht unwesentliche Abweichungen von dem gewöhnlich beobachteten Verlaufe der Erkrankung darbietet.

Bevor wir jedoch auf diese Abweichungen eingehen, wollen wir zuvörderst eine ausführliche Krankengeschichte des Falles geben.

Am Morgen des 5. Januar 1891 wurde ich zu dem Kaufmann, Herrn G., gerufen. Derselbe, ein 31 Jahre alter, kräftiger, untersetzter Mann mit stark entwickeltem Panniculus adiposus, welcher früher niemals an ernstlichen Erkrankungen gelitten hatte, klagte seit etwa 24 Stunden über heftige Schmerzen in der Gegend des linken Hüftgelenkes, welche theils spontan theils, und zwar in erhöhtem Maassstabe bei Bewegungen auftraten. Di Untersuchung ergab, was am Hüftgelenke nicht oft der Fall ist, eine deutliche Schwellung des Gelenkes und eine leichte Röthung der Hautbedeckung also alle Zeichen der serösen Synovitis mit periarticulärem Oedem. Obgleich nur dies eine Gelenk befallen war, so stellte ich doch die Diagnose mi aller Sicherheit auf acute Polyarthritis. Hierzu bewog mich auch die anamnestische Angabe des Patienten, dass seine Stellung als Lagerverwalter ihn den Witterungseinflüssen in ganz besonderem Maasse aussetzte. - Eine Temperaturerhöhung wurde bei der ersten Untersuchung nicht gefunden. Patient erhielt stündlich $1 / 2 \mathrm{~g}$ Acidum salicylicum in Limousin'schen Kapseln, das Gelenk wurde sorgfältig in Watte gepackt und die entsprechende Diät angeordnet. Der weitere Verlauf der Krankheit war der eines gewöhnlichen schweren Falles ron acutem Gelenkrheumatismus. 'Iäglich wurden 1 oder 2 Gelenke von neuem befallen, besonders stark die Knieund Schultergelenke. Es wurde späterhin statt der Salicylsäure salicylsaures Natron (10:200) 2 stündlich ein Esslöffel voll gereicht, und die Gelenke wurden mit Collodium bestrichen. Die Temperatur überstieg in der ganzen Zeit nicht 390. Auffallend war die absolute Schlaflosigkeit des von Schmerzen nur sehr wenig geplagten Patienten, die weder durch kleine Dosen Morphium, noch durch Chloralhydrat wirksam bekampft werden konnte. Bis hierher bietet der Fall keinerlei Besonderheiten. Jedoch am 12. Januar, genau eine Woche nach Beginn des Leidens, trat eine Wendung in demselben ein. Nachdem ich noch am Morgen dieses Tages den Patienten welcher fast schmerzlos war und eine Temperatur von 38,4 gezeigt hatte, mit der Bemerkung verlassen hatte, dass ich an diesem Tage den Abendbesuch aussetzen würde, wurde ich plötzlich des Nachmittags 5 Uhr zu demselben gerufen, weil das eingelegte Thermometer $40^{\circ}$ zeige, und die Athmung eine sehr schlechte geworden sei. Ich fand den Patienten stark schwitzend und seh oberflächlich athmend (ca. $40 \mathrm{mal}$ in der Minute) im Bette liegen ohne Delirien, obne Convulsionen, Tricmus oder irgendwelche andere motorische Reiz symptome. Die Temperatur betrug 40,1, der Puls 130 . Es war unter diesen Umständen kaum zweifelhaft, ${ }^{\star}$ dass die Krankheit in die hyperpyretische Form übergegangen sei. Bald sollte jeder Zweifel schwinden.

Um $10 \mathrm{Uhr}$ abends hatte das Thermometer bereits $41^{\circ}$ erreicht, ohne dass jedoch das Bewusstsein des Patienten auch nur im geringsten Grade gestört worden wäre, oder sich irgend welche cerebrale Erscheinungen eingestellt hätten. Nur eine ausserordentliche Schwäche beunruhigte den Kranken, der sich eingehend erkundigte, ob sein Zustand Lebensgefahr be-

1) A rch. gén. de múd. 1862.

2) 1. c.

-) Orvosi hetilap. 1888, No. 23

${ }^{4}$ Zeitschr. f. Ohrenheilh. XVlll, p. 161 .

5) Deutsch. med. Wochenschr. 1890, No. 1\%. Ueber Otitis med. acuta nach Influenza. Von L. Jankau.

6) Verhandlungen der otiatrischen Section der Naturforscherversammlung in Wiesbaden. 1873 fürchten liesse. Ich beruhigte, wenn ich auch von einem baldigen tödtlichen Ausgang üherzeugt war den Patienten und ordnete eine Eisblase auf den Kopf, Einhüllungen des Körpers in kalte nasse Tücher, sowie öfteres Einflössen schweren Weines an. Sodann verliess ich das Haus mit der Mahnung, mich bei Verschlimmerung des Zustandes rufen zu lassen. Dies geschah etwa 1/21 Uhr des Nachts, Als ich den Kranken sah, hatte er bereits das Bewusstsein verloren. Auf Befragen erfuhr ich, dass er noch $1 / 4$ Stunde vorher nicht allein an ihn gerichtete Fragen deutlich und verständig beantwortet, sondern auch selbst kleine Gespräche geführt hatte. Er, der früher stets ausserordentlich stark geschwitzt hatte, strahlte jetzt eine trockene Hitze aus, dabei war jedoch das Gesicht blau, die Augen trühe, der Athem ausserordentlich frequent (45 in der Minute), öfter stertorös. Das eingelegte Thermometer zeigte nach 15 Minuten in der Achselhöhle 42,7, nacl ferneren 10 Minuten 43,6, und stieg so bis zum 'Tode, der um $1 \frac{1}{2}$ Uhr erfolgte, bis auf 44,2 . Ich versăumte nicht, den umstehenden Laien diese gewaltige Temperaturerhöhung zu demonstriren. Der Puls war deutlich fühlbar, setzte bei jeder Respiration aus und betrug trotzdem ca. 180 in der Minute. Allerdings war mir eine genaue Zählung desselben nicht möglich. Der 'lod trat ohne jeden Kampf ein.

Es ist nicht die ausserordentlich hohe Temperatur von $44,2^{\circ} \mathrm{C}$ in erster Linie, warum mir der vorliegende Fall mittheilungswerth erschiell. Denll ill der Litteratur existiren eine ganze Anzahl guter Beobachtungen, welche beim hyperpyretischen Gelenkrheumatismus, wenn auch nicht gleich hohe, so doch recht bedeutende Temperaturgrade vor dem Tode constatiren. So theilt beispielsweise Th. Simon einen Fall von Rheumarthritis mit, bei welchem derselbe eine Temperatursteigerung von $43,0^{\circ} \mathrm{C}$ beobaclitete. Unter gleichen Verhältnissen fand $\mathrm{S}$. Binger sogar $43,9^{\circ} \mathrm{C}$. Von anderen Beobachtern sei noch H. Immermann erwähnt, welcher in der Agone eines Kranken mit acutem Gelenkrheumatisinus eine terminale Hyperpyrexie vou $42,8^{\circ} \mathrm{C}$ constatirte. Ferner H. Weber und H. F. A. Goodridge, welche 2 Fälle von Polyarthritis rheumatica mit complicirender Endocarditis behandelten, in deren Verlaufe die Temporatur bis $109,5^{\circ} \mathrm{F}$ stieg. Aehnlich sind die beiden Krankheitsgeschichten von Barkley, 2 tödtlich verlaufende Fälle betreffend, welche vor dem Exitus eine Temperatur bis $43,8^{\circ} \mathrm{C}$ aufwiesen. Bei der Section wurde nichts besonderes nachgewiesen. Wir beschliessen die Aufzählung einschlägiger Fälle mit der Publication von Sidney Ringer. Derselbe beobachtete 4 Fälle von acutem Gelenkrheumatismus, welche bei anfangs gutartigem Verlaufe plötzlich Temperatursteigerungen von $42,8-43,9^{\circ} \mathrm{C}$ aufwiesen. Nach kurzdauernden Delirien trat in tiefem Sopor der Tod ein. Der ganze Verlauf der Hyperpyrexie betrug $1^{1 / 2}-16$ Stunden. Von diesen 4 Fällen waren 3 mit Herzaffectionen complicirt, und zwar der eine mit Pericarditis, die beiden anderen mit Endocarditis. Das Alter der Patienten schwankte zwischell 20-29 Jahren. Im Anschluss hieran sei noch bemerkt, dass Quincke ill einem Falle von acuter Polyarthritis rheumatica eine postmortale Temperatursteigerung bis auf $43,95^{\circ} \mathrm{C}$ in der Axilla und $44,7^{\circ} \mathrm{C}$ in der Vagina, und zwas. 40 Minuten post mortem beobachtet hat. Wir sehen also, dass in keinem der angeführtell Fälle intra vitam einc gleich hohe Temperatur $(44,2)$, wie in unserem Falle, beobachtet wurde. Sieht mall jedoch vom Rheumatismus articulorum acutus ab, so kann man bei gewissen anderen Krankheitszuständen ähnlich hohe und noch ungleich höhere, kaum glaubhaft klingende Temperatursteigerungen beobachten. So berichtet Law son Tait über einen Fall von doppelseitiger Ovariectomie, nach welcher sich plötzlich eine Steigerung der Ejgenwärme bis auf $43,9^{\circ} \mathrm{C}$ bei 120 Pulsen einstellte. Am nächsten Morgen war die Temperatur bereits spontan auf $38,6^{\circ} \mathrm{C}$ gefallen. Es folgte eine schnelle Reconvalescenz. Die Messungen wurden mit drei verschiedenen Thermometern vorgenommen. Rivington theilt folgende Krankengeschichte mit. Eine 42 Jahre alte Frau, der man den linken Unterschenkel amputirt hatte, erholte sich nach diesem Eingriffe niemals vollkommen. Ein Jahr nach der Operation stellte sich am Amputationsstumpfe ein Erysipel ein, dazu Husten und leichtes Fieber. Plötzlich trat unter Frost und Hitze eine rechtsseitige Unterlappenpneumonie ein, in deren Verlaufe eine 'Temperatursteigerung bis 44,1 beobachtet wurde. Am auffallendsten hierbei war der Umstand, dass sich die Patientin hierbei im allgemeinen leidlich wohl befand. W. S. Brine behandelte eine 42 jährige Hysterica, welche früher an acutem Gelenkrheumatismus gelitten hatte. Dieselbe klagte lange Zeit über Schmerzen im Hypochondrium, ohne dass auch nur eine objective Erscheinung daselbst nachzuweisen war. Plötzlich hob sich die Körpertemperatur auf $114^{\circ} \mathrm{F}$ in der Achselhöhle. Der fieberhafte Zustand, in dessen Verlaufe das Thermometer als höchsten Stand $46,6^{\circ} \mathrm{C}$ erreichte, währte 11 Tage lang. Der Puls betrug in maximo 156 Schläge. Busch publicirt einen Fall von Mammacarcinom bei einer 42 Jakre alten Frau, nach dessen Entfernung eine plötzliche Temperatursteigerung von $43,3^{0}$ den Tod herbeiführte. H. Quincke sah nach einer Zerquetschung des Halsmarkes kurz vor dem Tode $43,8^{\circ} \mathrm{C}$ im Rectum. G. H. Philipson berichtet über ein 23 jähriges hysterisches Mädchen, welches über Brust- und Rückenschmerzen klagte und plötzlich eines Abends ohne sichtbare Veranlassung eine Temperatur von $44,40 \mathrm{C}$ in der 
Achselhöhle aufwies. Nach einigen Tagen, während deren die Körperwärme wiederum erheblich gesunken war, wurde in der linken Achselhöhle 47,20 C, in der rechten 43,30 C und in der Mundhöhle $14,4^{0} \mathrm{C}$ gemessen. Dabei war ein deutlicher Einfluss auf Sensorium Ind Nervensystem nicht nachzuweisen. Puls und Athmung blieben ohne Veränderung. Auch durch dauernde Verstopfung tritt zuweilen eine Erhölıung der Körpertemperatur um 2-30 C auf, wie dies Brüninghausen und $\mathrm{J}$. Schwering sell. mitgetheilt haben. Das ungewöhulichste jedoch, was die Litteratur ïber enorme Steigerung der Eigenwärme beim Menschen mittheilt, ist der von Senator im Centralblatt für die medicinischen Wissenschaften 1875 referirte Fall von L. W. Teale. Derselbe betrifft eine junge Dame, welche sich durch einen Sturz vom Pferde nicht unerheblich verletzte. Die Temperatur betrug Anfangs $101^{\circ} \mathrm{F}$ und wurde nach 14 Tagen wieder normal. Bald jedoch, unter Schmerzen im 6. Brustwirbel und leichten Zuckungen in den Schenkeln, stieg die Temperatur wiederum auf $101^{\circ} \mathrm{F}$, um dann binnen kurzer Zeit, etwa 2 Wochen, die enorme Höhe von $1220 \mathrm{~F}\left(50^{\circ} \mathrm{C}\right) \mathrm{zu}$ erreichen. Der Puls betrug dabei 120 Schläge und wurde filiform. 8 Tage lang etwa blieb die Temperatur gleich hoch, um dann allmählich abzufallen. Auf die das Fieber begleitenden Nebenumstände wollen wir an diesem Orte nicht eingehen. Es sei noch ausdrücklich bemerkt, dass zu den Messungen 7 Thermometer, von denen 4 in Kew controllirt worden waren, beuntzt wurden. Sechs dieser Thermometer besassen nur eine Scala bis $118^{\circ} \mathrm{F}\left(47,8^{\circ} \mathrm{C}\right)$, nur eins eine solche von $122^{\circ} \mathrm{F}$. Tetztere wurde an einem Tage noch etwa um 30 iiberschritten, sodass die Temperatur zum mindesten $51,65^{\circ} \mathrm{C}$ betragen haben musste.

Wichtiger als die colossale, bei hyperpyretischem Gelenkrlıeumatismus bisher noch niemals beobachtete Temperatur von $44,2^{\circ} \mathrm{C}$ in meinem Falle, ist ein anderer Umstand, der gleichfalls, soweit meine Kenntniss reicht, noch niemals beobachtet oder doch wenigstens niemals publicirt worden ist. Es ist dies die auffallende Erscheinung, dass trotz und während der hohen Steigerung der Körperwärme das Sensorium des Patienten bis $1^{1 / 2}$ Stunde vor dessen Tode völlig ungetrübt blieb, so ungetrïbt, dass seine Umgebung, mit welcher er noch bis zu dem mitgetheilten Zeitpunkte verständig und verständlich sprach, an die Möglichkeit eines ungünstigen Ausganges nicht $\mathrm{zu}$ denken wagte.

Die bislang beobachteten Fälle von hyperpyretischem Gelenkrheumatismus zeigten stets eine Reihe cerebraler Symptome, die in dem Grade das ganze Bild der Krankheit beherrschten, dass man auch schlechtweg von "Cerebralrheumatismus" sprach. Schon in der ersten Zeit vor Beginn der hohen Temperaturen pflegten die Kranken neben ausgeprägter Schlaflosigkeit an leichten Delirien zu leiden, die sich meist des Abends einstellten. Sobald aber das hyperpyretische Stadium einsetzt, beginnen sich in acutester Form die schwersten Cerebralsymptome zu entwickeln. Neben heftiger Unruhe und Delirien hat man auch öfters motorische Reizerscheinungen, allgemeine Convulsionen, Trismus und tonische Starrheit der Glieder beobachtet. Von alledem war bei meinem Patienten keine Rede. Das einzige in dieser Hinsicht bemerkenswerthe war eime allerdings auffallend lange anhaltende Schlaflosigkeit des Kranken, die durch mässige Gaben von Morphium und Chloralhydrat nicht erheblich beeinflusst werden konnte. Hiervon abgesehen, konnten absolut keine Gehirnerscheinungen beobach tet werden. Als das Thermometer $410 \mathrm{C}$ bereits überschritten liatte, besprach der Patient noch in aller Ruhe seine Erkrankung, und als ich des Nachts um 12/2 Uhr gerufen wurde und eine Temperatursteigerung von nicht weniger als $42,70 \mathrm{C}$ constatirte, erfuhr ich, dass etwa erst $1 / 4$ Stunde vor meiner Ankunft der Kranke das Bewusstsein verloren, nachdem er bis dahin noch ganz verständige Reden gefülırt hatte.

Bezüglich der Ursachen jener plötzlichen Temperaturerhöhungen stehen wir noch vor einem Räthsel. Im vorliegenden Falle handelte es sich um einen dem Alkohol in verschiedenster Gestalt nicht abholden Patienten, und so ist derselbe ein Beweis mehr für die Aunahme Senator's, dass in der Regel Potatoren von dem in Rede stehenden Leidén befallen werden. Strümpell kann dies durch seine eigenen Erfahrungen nicht bestätigen; er nimmt vielmehr an, dass kein einziger Fall vor dem Eintreten des hyperpyretischen Stadiums gesichert sei.

Wir wissen, dass das Hauptsymptom jenes Zustandes, den wir Fieber nennen, eine Erhöhung der Körpertemperatur darstellt; wir wissen ferner, dass diese letztere im wesentlichen auf einem Missverhältniss zwischen Wärmeproduction und Wärmeabgabe beruht. Die Verringerung der Wärmeabgabe nach aussen scheint, wie ich dies in meiner Arbeit ,Calorimetrische Untersuchungen über die Wärmeproduction und Wärmeabgabe des Armes am Gesunden und Kranken", Archiv für Anatomie und Physiologie (Physiologische Abtheilung), 1887 ausgeführt habe, in dieser Hinsicht in erster Linie verantwortlich gemacht werden $z u$ müssen. Diese Verringerung kommt dadurch zustande, dass jenes uns noch unbekannte, das Fieber erregende Agens eine specifische Wirkung auf die Vasomotorencentra ausübt, und zwar entweder in der Weise, dass es die Vasoconstrictoren erregt und durch die nun entstehende Verengerung der Gefässe die Wärmeabgabe nach aussen verringert, oder aber in der Weise, dass es die Vasodilatatoren unempfindlich macht, wodurch derselbe Erfolg erzielt wird. Vielleicht handelt es sich in den Fällen von Hyperpyrese um eine besonders schwere, plötzlich sich verstärkende Infection mit jenem fiebererregenden Agens, oder es sind die wärmeregulirenden Centra in der Medulla oblongata und im Rückenmark bei diesen Kranken in ganz besonderem Maasse reizbar.

Zum Schlusse sei noch bemerkt, dass die Messungen mit zwei Thermometern ausgeführt wurdeu, welche mit einem Normalthermometer verglichen wurden. Für die Praxis ergiebt sich die Nothwendigkeit, die Scala der gewöhnlich nur bis $44^{\circ} \mathrm{C}$ bestehenden ärztlichen Thermometer noch zu erweitern. 